\title{
Functional magnetic resonance imaging using RASER
}

\author{
Ute Goerke ${ }^{\mathrm{a}}$, Michael Garwood ${ }^{\mathrm{a}}$, and Kamil Ugurbila \\ ${ }^{a}$ Center for Magnetic Resonance Research and Department of Radiology, University of Minnesota, \\ 2021 6th St. SE, Minneapolis, Minnesota 55455, USA
}

\begin{abstract}
Although functional imaging of neuronal activity by magnetic resonance imaging (fMRI) has become the primary methodology employed in studying the brain, significant portions of the brain are inaccessible by this methodology due to its sensitivity to macroscopic magnetic field inhomogeneities induced near air filled cavities in the head. In this paper, we demonstrate that this sensitivity is eliminated by a novel pulse sequence, RASER (rapid acquisition by sequential excitation and refocusing) (Chamberlain et al., 2007), that can generate functional maps. This is accomplished because RASER acquired signals are purely and perfectly $T_{2}$ weighted, without any $T_{2}{ }^{*}$-effects that are inherent in the other image acquisition schemes employed to date. $T_{2}$-weighted fMRI sequences are also more specific to the site of neuronal activity at ultrahigh magnetic fields than $T_{2}{ }^{*}$-variations since they are dominated by signal components originating from the tissue in the capillary bed. The RASER based fMRI response is quantified; it is shown to have inherently less noisy time series and to provide fMRI in brain regions, such as the orbitofrontal cortex, which are challenging to image with conventional techniques.
\end{abstract}

\section{Keywords}

Functional magnetic resonance imaging; orbitofrontal cortex; susceptibility artifacts; Stroop test; visual cortex; ultrahigh magnetic field

\section{Introduction}

Functional magnetic resonance imaging (fMRI) is the most commonly employed technique for probing human brain function. However, fMRI has failed to provide clear access to regions of the orbitofrontal cortex and the temporal lobes, due to close proximity to air filled cavities and consequent inhomogeneities in static magnetic field magnitude. This limitation has been one of the most significant shortcomings of fMRI in studying human cognition. In this paper, we present a method that eliminates this limitation.

In fMRI, image contrast is based on physiologic and metabolic responses evoked by alterations in neuronal activity. The most commonly employed fMRI strategy is based on a gradient echo (GE) sequence where alteration in neuronal activity is encoded as a change in the relaxation parameter $T_{2}^{*}$ using a simple delay between spin excitation and commencement of spatial-

\footnotetext{
(c) 2010 Elsevier Inc. All rights reserved.

Corresponding author: Ute Goerke, PhD, Center for Magnetic Resonance Research, University of Minnesota, $20216^{\text {th }}$ St SE, Minneapolis, MN 55455, USA, Phone: 612-626-2001, Fax: 612-626-2004, ute@ cmrr.umn.edu.

Publisher's Disclaimer: This is a PDF file of an unedited manuscript that has been accepted for publication. As a service to our customers we are providing this early version of the manuscript. The manuscript will undergo copyediting, typesetting, and review of the resulting proof before it is published in its final citable form. Please note that during the production process errors may be discovered which could affect the content, and all legal disclaimers that apply to the journal pertain.
} 
encoding for the image. The latter is typically based on an ultrafast imaging sequence, such as EPI or SPIRAL techniques.

In areas of the brain near air-filled cavities the magnetic field is highly non-uniform because of the magnetic susceptibility difference between the air space and tissue. Consequently, in these regions, the effective $T_{2} *$ is much shorter than spatial encoding periods currently achievable for EPI or SPIRAL. Functional contrast imposed as a simple delay between signal excitation and image acquisition (i.e. GE contrast) exacerbates the situation. As a result, signal dropout and image distortions or blurring are often observed in the orbitofrontal lobe, inferior temporal lobe, and basal regions of the brain. These problems become worse at ultra-high magnetic fields. Considerable effort has been invested to reduce such effects. The z-shim method requires additional data acquisition (Constable and Spencer, 1999, Du et al., 2007, Glover, 1999, Gu et al., 2002, Heberlein and Hu, 2004, Takahashi et al., 2001). Use of passive shims does not fully eliminate magnetic field variations (Cusack et al., 2005, Juchem et al., 2006, Koch et al., 2006, Wilson et al., 2003, Wilson and Jezzard, 2003, Yang et al., 2006). Parallel imaging alleviates the problem, but does not eliminate it completely (Bellgowan et al., 2006, de Zwart et al., 2006, Golay et al., 2004, Lin et al., 2005, Moeller et al., 2006, Preibisch et al., 2003, Pruessmann et al., 1999).

Signal loss that occurs during the post-excitation delay in GE-fMRI can be partially recovered with a "refocusing" RF pulse. The spin-echo (SE) that is formed retains sensitivity, albeit a smaller one, to alterations in neuronal activity. This functional contrast arises from diffusioninduced dynamic averaging of magnetic field gradients near capillaries and venules in the extravascular space, and intravascular changes of the effective $T_{2}$ of blood. Although inherently weak, because of a supralinear dependence on the static magnetic field magnitude, the SE fMRI becomes robustly detectable at ultrahigh magnetic fields (e.g. (Ugurbil et al., 1999, Ugurbil et al., 2003, Yacoub et al., 2007, Yacoub et al., 2008) and references therein). Furthermore, it has the advantage of higher specificity to the site of neuronal activation at ultrahigh magnetic fields (e.g. (Ugurbil et al., 1999, Ugurbil et al., 2003, Yacoub et al., 2007, Yacoub et al., 2008) and references therein).

Although SE fMRI avoids sensitivity to macroscopic magnetic field inhomogeneities during the contrast preparation, when the ensuing spatial encoding is performed by EPI or SPIRAL strategies (i.e. SE-EPI or SE-SPIRAL, respectively) the resulting image still suffers from spurious $T_{2}{ }^{*}$-contributions (Birn and Bandettini, 2002). Only the echo in the center of the $\boldsymbol{k}$ space is acquired at the nominal echo time and is purely $T_{2}$-weighted. As a consequence, less accurate (i.e. GE-like) contributions contaminate the fMRI data; more importantly, signal attenuation, often to below detection limits, persist in the reconstructed images in regions of macroscopic susceptibility variations. The $T_{2}{ }^{*}$-weighting also results in image blurring since the decreasing echo amplitude (from $T_{2}{ }^{*}$-decay) is equivalent to an apodization filter. Signal dropout is completely eliminated in images acquired with fast spin-echo (FSE) sequences using a long preparation delay for $T_{2}$-weighting and short refocusing intervals for spatial encoding (Poser et al., 2006, Poser and Norris, 2007). A confound of these techniques is, that $T_{2^{-}}$ weighting varies as a function of spatial phase-encoding. Hence, the echo train has to be kept short. In addition, at high fields, where fMRI contrast and accuracy improves, power deposition becomes a limiting constraint for FSE sequences.

When magnetic field inhomogeneities are not large enough to lead to complete signal dropout, they still degrade images by causing geometric distortions in EPI. These geometric distortions depend on the amplitude of the susceptibility-induced macroscopic gradient relative to the imaging gradients used for spatial encoding. EPI is especially susceptible to geometric distortion in the direction of the phase-encoding gradient since these gradient blips are typically two orders of magnitude smaller than the gradient in the readout or the slice-selection direction. 
Several techniques have been implemented to correct for image distortions (Chen and Wyrwicz, 2001, Cordes et al., 2000, Liu and Ogawa, 2006, Ojemann et al., 1997). In addition, improved local $B_{0}$-shimming approaches reduce geometric distortions of the image (Constable and Spencer, 1999, Cusack et al., 2005, Du et al., 2007, Glover, 1999, Gu et al., 2002, Heberlein and $\mathrm{Hu}, 2004$, Juchem et al., 2006, Koch et al., 2006, Takahashi et al., 2001, Wilson et al., 2003, Wilson and Jezzard, 2003, Yang et al., 2006). Most of these techniques have been shown to be successful to some degree but not fully, and require significant effort and additional imaging time for careful calibration and optimization.

Recently, we introduced a novel $T_{2}$-weighted sequence, rapid acquisition by sequential excitation and refocusing (RASER) (Chamberlain et al., 2007). In this sequence, all echoes are acquired at exactly the same echo time. Hence, the signal has the same $T_{2}$-weighting independent of spatial encoding, eliminating the artifacts and limitations of previously described techniques. In addition, phase-encoding is replaced by time-encoding in RASER, which uses larger encoding gradients. Therefore, image distortions are minimal. In this paper, we demonstrate the first use of RASER for fMRI of human brain. The statistical significance of brain activation is quantified and compared with GE-EPI and SE-EPI. Examples of brain regions, such as the orbitofrontal lobe, which are beyond the reach of conventional imaging methods, are shown.

\section{Materials and Methods}

\subsection{Overview of fMRI experiments}

A variety of fMRI experiments were performed in order to demonstrate the feasibility and robustness of detecting the vascular response using RASER. An overview of paradigms, imaging parameters and number of subjects is listed in table 1. The Stroop color-word match test was used to engage the orbitofrontal cortex (OFC). In the first part of the project, the impact of B0-inhomogeneties, which are caused by the proximity of OFC to the air-filled sinuses, on the degree of image artifacts and the quality of functional activation maps is demonstrated. To this end, Stroop test studies were performed comparing GE-EPI, SE-EPI and RASER for a variety of imaging parameters and paradigm conditions. In a first set of experiments, axial slices with a moderate spatial resolution of $(3 \mathrm{~mm})^{3}$, which is typically used in $3 \mathrm{~T}$ fMRI experiments with whole brain coverage, were acquired. To detect a robust signal change, incongruent versus neutral and congruent versus neutral condition of Stroop test was mapped. In a second set of experiments using the same imaging parameters, the minute signal change difference between the incongruent and congruent condition of the Stroop paradigm are probed in order to provide a qualitative measure of detection sensitivity. A third set of Stroop fMRI experiments were performed in order to compare activation maps between EPI-based methods and RASER with imaging parameters, which are more favorable for EPI in the OFC at $7 \mathrm{~T}$. For this set of experiments, coronal slices with high spatial resolution $\left(1.5 \times 1.5 \times 2 \mathrm{~mm}^{3}\right)$ were acquired (Deichmann et al., 2003).

In addition to the qualitative assessment of detecting the BOLD response using RASER in comparison with the EPI-based imaging methods, visual stimulation studies were performed. The visual stimulation paradigm provides sufficiently robust BOLD signal changes, which to be able statistically analyze the contrast-to-noise for the three imaging sequences. This analysis is used to explain the observed superior quality of RASER activation maps compared to the EPI-based imaging methods in the OFC.

\subsection{Hardware}

Experiments were performed on a $7 \mathrm{~T}$ Siemens scanner (Erlangen, Germany). A quadrature surface radio-frequency (RF) coil was used for the fMRI study of the visual cortex with noise 
analysis. Body gradients (maximal gradient amplitude: $40 \mathrm{mT} / \mathrm{m}$, a slew rate: $170 \mathrm{~T} / \mathrm{m} / \mathrm{s}$ ) were used for the visual stimulation study. The phantom experiment was performed with a 16channel volume RF head coil and a head gradient setup (maximal gradient amplitude: $80 \mathrm{mT} /$ $\mathrm{m}$, a slew rate: $400 \mathrm{~T} / \mathrm{m} / \mathrm{s}$ ). For the color-word match Stroop test fMRI study, the same volume RF head coil was utilized. One session for the Stroop-test study was performed with the body gradients, and for the remaining ones the head gradients were used. All imaging parameters for the fMRI scans with the Stroop paradigm were kept the same for the two gradient setups, except the gradient ramps were shorter with the head gradients. Paradigms were presented using goggles from Avotec, Inc (Stuart, Florida, USA) through an opening in the RF-coil.

\subsection{Subjects and psychophysical procedures}

Written voluntary consent was obtained from all subjects prior to all experiments. Five volunteers (two female) participated in the visual stimulation experiment. In the Stroop colorword match task studies, seven subjects (four female) in total took part (see table 1).

The color-word interference Stroop test was described in more detail in references (Treisman and Fearnley, 1969, Zysset et al., 2001). Briefly, it consists of word pairs, in which in the color of the upper word has to be matched with the meaning of the lower word printed in black. In the congruent condition the upper word was printed in the corresponding color, whereas in the incongruent condition it was printed in a noncorresponding color. The neutral condition consisted of replacing the upper word by the letters 'XXXX' printed in color. Each word pair was presented for $2 \mathrm{~s}$. The button responses of the volunteer corresponding to the answers 'YES' and 'NO' to the question, whether the meaning of the lower word matches the color of the upper word, were recorded. The volunteers had practiced the task several times and performed the test with ease and stability.

In the first Stroop test study, 10 blocks of $24 \mathrm{~s}$ of congruent, $24 \mathrm{~s}$ of incongruent and $24 \mathrm{~s}$ of the neutral condition was presented. At the beginning and the end of the scan, a $30 \mathrm{~s}$ of a black screen with a fixation cross was displayed. In the second Stroop test study with moderate spatial resolution with an axial slice orientation as well as the third Stroop study with high spatial resolution and coronal slice orientation, three blocks of $30 \mathrm{~s}$ of the congruent condition were alternated with $30 \mathrm{~s}$ of the incongruent condition. The imaging sequences were repeated three times in random order to avoid trends induced by habituation. One of two volunteers participated in the second study on three different days in order to determine the reproducibility of activation in the frontal cortex. In the third Stroop study with high spatial resolution and coronal slice orientation, two subjects performed the task.

In the visual stimulation experiment, five blocks of $30 \mathrm{~s}$ of a red circular checkerboard flashing at $8 \mathrm{~Hz}$ ("on") alternating with $30 \mathrm{~s}$ of black screen ("off") were presented. Subjects were asked to fixate a cross in the center of the screen. The imaging methods were performed in a randomized order in different subjects.

\subsection{MR imaging methods}

fMRI experiments were performed with three different sequences: RASER, SE- and GE-EPI. In the EPI-sequences, navigator-echoes were used to generate full reference scans to phasecorrect shifts of even and odd echoes of the images (Bruder et al., 1992). In all EPI-experiments, fat suppression was applied. Figure 1 shows the RASER sequence (Chamberlain et al., 2007). The excitation pulse is a chirp-pulse calibrated to a $90^{\circ}$ flip angle. During the frequencysweep of the chirp pulse an isochromat is rotated into the transverse plane when the sweepfrequency is equal to the spins' resonance frequency (Park and Garwood, 2009). If a static magnetic field gradient, $G_{t}$, is applied during the chirp-pulse the spatial dimension, $x_{t}$, is effectively encoded in time referred to as time-encoding indicated by the subscript $t$ 
(Chamberlain et al., 2007, Tal and Frydman, 2006). By using two slice-selective adiabatic hyperbolic secant pulses, the transverse magnetization is refocused at sequential times in the order in which it was excited by the frequency-swept chirp pulse. The second spatial dimension is frequency-encoded using alternating readout gradients, $G_{R}$, in analogy to EPI. A linear phasecorrection along the time-encoded dimension of echoes is applied according to reference (Chamberlain et al., 2007). RASER images are then obtained by 1D Fourier transformation for 2D-RASER.

The 3D hybrid version of RASER, previously referred to as multi-slice RASER emphasizing the "slice-selective" property in its time-encoded dimension, $x_{t}$, (Chamberlain et al., 2007) employs a phase-encoding gradient in the slab-selective dimension which is incremented in subsequent scans (gray shaded gradient pulses in Figure 1), that is, the two spatial dimensions, $x_{t}$ and $x_{R}$, are encoded in a single shot, while the third spatial dimension, $x_{s}$, is a multi-shot acquisition scheme. In multi-slice RASER, a second Fourier-transformation of the phaseencoded slab-selective dimension is performed. In RASER, no fat suppression pulse was needed since the signal displacement due to the chemical shift offset in the time-encoded dimension is only about two voxels.

In the two Stroop test studies, 8 axial slices covering the orbitofrontal cortex at a position at which activation was observed in reference (Norris et al., 2002) were selected. Multi-slice GEEPI and SE-EPI images were recorded single-shot with an $3 \times 3 \mathrm{~mm}^{2}$ in-plane resolution, $3 \mathrm{~mm}$ slice thickness, and a field-of-view of $192 \times 192 \mathrm{~mm}^{2}$. In RASER, 8 phase-encoding steps were acquired to encode the third spatial dimension with a spatial resolution of $3 \mathrm{~mm}$. For RASER the field-of-view was $192 \times 96 \mathrm{~mm}^{2}$ providing the same in-plane resolution as in the EPI images. The temporal resolution was $6 \mathrm{~s}$ rendering a shot-to-shot repetition time of $T_{R}=6 \mathrm{~s}$ for the EPI-sequences and $T_{R}=750 \mathrm{~ms}$ for RASER. The excitation flip angle was adjusted to nominal $90^{\circ}$ for the EPI-sequences and $45^{\circ}$ in RASER to account for saturation effects. The echo times were $25 \mathrm{~ms}$ for GE-EPI and $60 \mathrm{~ms}$ for SE-EPI and RASER. The acquisition bandwidth in readout direction was $2298 \mathrm{~Hz} /$ pixel. The time-bandwidth product of the chirp-pulse for excitation in RASER was $R=552$ and its pulse duration was $16.98 \mathrm{~ms}$. In the first study, 131 repetitions were acquired per functional time series. In the second Stroop test experiment, 41 repetitions were acquired in all functional scan.

In the third Stroop study, high resolution images were acquired. The in-plane resolution of the coronal slices was $1.5 \times 1.5 \mathrm{~mm}^{2}$ with a slice thickness of $2 \mathrm{~mm}$. For the two EPI sequences, 12 slices without gap were acquired. In order to be able to cover a matrix of $128 \times 128$ points without causing blurring due to $T_{2}{ }^{*}$-weighting along the phase-encoded dimension (Birn and Bandettini, 2002), four segments for each slice were acquired. In RASER, the FOV was zoomed in the time-encoded dimension to achieve a high in-plane resolution. The acquired matrix in RASER was $128 \times 24 \times 8$, where the third dimension was phase-encoded in subsequent scans with a reduction factor of 2 (half of the FOV) using parallel imaging and reconstructed using GRAPPA to obtain the unfolded image with a final matrix size of 16 voxels in third dimension (Griswold et al., 2002). The acquisition bandwidth in the readout direction was 2439 $\mathrm{Hz} /$ pixel. For RASER, the time-bandwidth product of the chirp-pulse was $R=412$ and its duration was $19.04 \mathrm{~ms}$. The excitation flip angle was adjusted to nominal $90^{\circ}$ for the EPIsequences and $45^{\circ}$ in RASER. The volume-to-volume repetition time was $6 \mathrm{~s}$ for all imaging methods resulting in shot-to-shot repetition time of $750 \mathrm{~ms}$ for RASER. The echo time $25 \mathrm{~ms}$ for GE-EPI and $60 \mathrm{~ms}$ for SE-EPI and RASER. 40 repetitions were acquired for all functional scans. Signal instabilities due slight phase errors between subsequent scans were not corrected for. Since all three sequences are based on multi-shot acquisitions schemes image quality of all imaging methods are affected by physiological noise in the spatial dimension, which is encoded in multiple scans. 
In the visual stimulation study, a single oblique slice along the calcarine sulcus was selected. For 2D RASER, SE-EPI, and GE-EPI, the imaging parameters were $2 \times 2 \mathrm{~mm}^{2}$ in-plane resolution, slice thickness: $3 \mathrm{~mm}$, FOV: $128 \times 64 \mathrm{~mm}^{2}$, repetition time: $2 \mathrm{~s}$, readout bandwidth: $1698 \mathrm{~Hz} / \mathrm{pixel}$, and a nominal excitation pulse of $90^{\circ}$. The echo times were $25 \mathrm{~ms}$ for GE-EPI and $66 \mathrm{~ms}$ for SE-EPI and RASER. 166 repetitions were acquired in each functional experiment. The bandwidth time product for the chirp-pulse for excitation in RASER was $R=$ 552 and the pulse duration was $21.798 \mathrm{~ms}$. Resting state data (baseline scan), in which the subjects were asked to refrain from any movement and in which no stimulation was presented, were acquired with the same imaging parameters as in the visual stimulation experiment. A second scan (noise scan) was acquired without RF-pulses. 64 repetitions were recorded in the baseline and the noise scans.

$T_{1}$-weighted anatomical images were acquired for each fMRI data set. In order to avoid another post processing step to register anatomical and functional data, imaging parameters, such inplane resolution, thickness and slice position, of the anatomical images were matched with parameters of the functional scans. In the first two studies with the Stroop test, high-resolution inversion recovery 3D GRE images with an isotropic resolution of $1 \mathrm{~mm}$ covering a field-ofview of $256 \times 192 \times 144 \mathrm{~mm}^{3}$ were acquired. In the third Stroop test study and visual stimulation study, a single-slice inversion-recovery FLASH sequence was used to obtain an anatomical image of the OFC and primary visual cortex, respectively. The in-plane resolution in the OFC was $1.5 \mathrm{~mm}$ and in the primary visual cortex $1.0 \mathrm{~mm}$. The field-of-view and the slice thickness were matched with the respective functional images. For Figure 6, images were interpolated from a spatial resolution of $1.5 \times 1.5 \times 2 \mathrm{~mm}^{3}$ to an isotropic voxel size of $(1.5 \mathrm{~mm})^{3}$. In all $T_{1^{-}}$ weighted anatomical imaging scans, the inversion time was $1.5 \mathrm{~s}$

\subsection{Analysis of the functional time-series}

In all figures, the anatomical images as well as images obtained from functional time series, strong variations in signal intensity due to the large $B_{1}$-inhomogeneity at ultrahigh magnetic field were eliminated by normalizing images with a coil profile reference image. The reference image was obtained by smoothing the average image with a spatial Gaussian filter with a halfwidth-half-maximum of 20 voxels to eliminate the contrast related to anatomical structures of the brain.

To map activation, the $t$-scores represent the statistical significance of the mean signal difference in a time series between two conditions is calculated voxel-by-voxel using Stimulate (Strupp, 1996). Image reconstruction of the time series and further statistical analysis were performed with programs written in IDL (Bolder, Colorado, USA) or matlab (The MathWorks Inc., Natick, Massachusetts, USA).

For the Stroop test studies, the signal from the scalp and muscle, which generate false positives due to high physiological noise in the $t$-maps, were masked. Functional scans, which were acquired with the same sequence and parameters in the same session, were averaged. Before further analysis, data were spatially smoothed with a Gaussian filter with half-width-halfmaximum of two voxels. The time series from the first Stroop study were temporally filtered with a digital bandpass filter with cut-off frequencies of 0.0035 and $0.0833 \mathrm{~Hz}$ and a filter order of 20. The cut-off frequencies for temporal filtering of the data of the second were 0.0125 and $0.125 \mathrm{~Hz}$ and for third study 0.0083 and $0.083 \mathrm{~Hz}$ (filter order 20). For the first Stroop experiments, $t$-maps of congruent versus neutral and incongruent versus neutral condition were computed. The second and third study provided $t$-maps representing incongruent versus congruent condition. All $t$-maps were zero-filled appropriately to match the corresponding high-resolution anatomy and smoothed with a spatial Gaussian filter with a half-width-halfmaximum of two voxels. 
In Figure 2 and 5, the time averaged images of the corresponding time series, which were acquired with RASER, GE-EPI, and SE-EPI, are shown. Figure 3 shows $t$-maps corresponding to congruent or incongruent versus neutral condition are superimposed on the high-resolution $T_{1}$-weighted anatomy. In the second Stroop study, $t$-maps for incongruent versus congruent condition, which were obtained from the same subject on three different days, were aligned to one high-resolution anatomy, summed voxel-by-voxel, and divided by $\sqrt{N}$, where $\mathrm{N}=3$ is the number of added $t$-maps (Figure 4) (Bosch, 2000). Regions with significant signal changes related to the paradigm in the anterior part of the frontal cortex are listed in Table 2. In Figure $6, t$-maps of the study with high spatial resolution and a coronal slice orientation are shown. The $t$-score represents the statistical significance of differences in the BOLD-response of the incongruent versus congruent condition.

For the visual stimulation study, $t$-maps representing the "on" (flashing checkerboard) and the "off" (black background) condition were calculated from the time-courses acquired with RASER, SE-EPI, and GE-EPI. Thresholded $t$-scores representing statistical significance of the mean difference between the on and the off-conditions are superimposed on the images (Figure 7). The displayed images were zero-filled to twice the matrix size and smoothed with Gaussian filter with a half-maximum half-width of 1.5 voxels.

Regions-of-interest (ROIs) were selected by thresholding the $t$-maps of the activation detected in the primary visual cortex. For each subject, the thresholds for the GE- and SE-EPI $t$-maps were adjusted to select the same number of activated voxels as in the corresponding RASER $t$-maps, in which the threshold was set to $t=2.5$ (Figure 7). These voxel clusters defined intermediate ROIs for each imaging method. For each subject, the reference ROI, on which the statistical analysis was based, was generated by combining the intermediate ROIs obtained for each imaging sequence.

Figure 8 shows the mean trial averages of all subjects representing the average of all stimulation blocks of all voxel time courses within the reference ROI in the primary visual cortex. Time courses of activated voxels in a region-of-interest (ROI) in the visual cortex were averaged over all subjects $(\mathrm{N}=5)$. The error bars represent the standard deviation of the variation between subjects. The maximal relative signal change, $\Delta \mathrm{S} / \mathrm{S}$, in Table 3 was calculated from the mean between $11 \mathrm{~s}$ and $29 \mathrm{~s}$ after stimulus onset of the trial averages. The standard error (mean of the error in trial averages divided by the square root of the number of included time points) is listed. Furthermore, the mean $t$-scores, $t_{\text {average, }}$, of the ROI averaged over all subjects were computed (Table 3). The error represents the standard deviation of variations between subjects.

The ratio of the standard deviation of the physiological noise over the standard deviation of the intrinsic noise, $\sigma_{\text {phys }} / \sigma_{\text {intr }}$, was calculated from ROI voxel-by-voxel from the baseline and the noise scan. The signal-to-noise ratio, SNR, represents the ratio of the standard deviation of the physiological noise over the signal intensity of time courses of the baseline data within the ROI previously defined. The mean and the standard deviation of the group of subjects for $\sigma_{\text {phys }} / \sigma_{\text {intr }}$ and SNR are listed in Table 3 . The contrast-to-noise ratio, CNR, is calculated from the product of the maximal relative signal change, $\Delta \mathrm{S} / \mathrm{S}$, and the signal-to-noise ratio, SNR. The error for CNR is derived through error propagation.

\section{Results}

\section{1 fMRI in the orbitofrontal cortex using multi-slice RASER}

Three studies were performed to demonstrate the differences of image artifacts and the quality of activation maps in the OFC, obtained with GE-EPI, SE-EPI, and RASER fMRI. Figure 2 shows one axial slice out of eight obtained from the fMRI time series acquired with GE-EPI, SE-EPI and RASER. To keep the echo time and, hence, the $T_{2}$-weighting of RASER consistent 
with SE-EPI ( $T_{E}=60 \mathrm{~ms}$ ) only 32 time-encoding steps instead 64 phase-encoding steps as in the EPI-sequences were acquired. Hence, only half of the field-of-view of EPI is covered with RASER. In RASER, the signal excitation is confined to the bandwidth of the excitation chirp pulse along the time-encoded dimension and, therefore, no transverse magnetization outside the FOV is generated. RASER can therefore zoom in on a region-of-interest without aliased signal from outside the FOV. In Figure 2, the EPI images exhibit severe signal loss and image distortions due to the sensitivity of phase-encoding with gradient blips to $B_{0}$-inhomogeneities. RASER images reveal neither signal loss nor distortions in the presence of magnetic field inhomogeneities. Therefore, it provides a solution to functional imaging in the regions of the brain that are not easily accessible with existing fMRI approaches, especially at ultrahigh magnetic fields.

This capability is illustrated in this work using the color-word-match Stroop test (Norris et al., 2002) that is expected to engage the orbitofrontal cortex (OFC). In Figure 3, thresholded activation maps are superimposed on the corresponding $T_{1}$-weighted anatomical images. Only the maps obtained from the RASER time series show significant signal changes, which correlate with the congruent versus neutral (middle column) and incongruent versus neutral condition (right column). Differences of the $t$-score for the two conditions are expected to be slightly different since the two conditions engage the executive brain regions to a different extent. The $t$-maps of the second volunteer were similar to the ones shown in Figure 3.

The second study was aimed at demonstrating the reproducibility of minute differences of the fMRI signal of the incongruent versus congruent condition of the word-color match Stroop test in the frontal lobe using RASER. To avoid registration of brain anatomies of different volunteers, the same subject participated in this study on three different days. Corresponding maps obtained in these three sessions were registered to the same high-resolution anatomy and averaged. RASER yielded significant activation in a variety of brain regions in the frontal lobe (Figure 4 and Table 2). Similar activated regions were found in a previous $3 \mathrm{~T}$ study using the color-word match Stroop task, utilizing SE-EPI optimized for minimal image distortions in the OFC (Norris et al., 2002). However, the previous study required averaging across seven volunteers, in contrast to averaging the maps obtained in three sessions (Figure 3) even though it was conducted under the less challenging conditions of $3 \mathrm{~T}$ where magnetic field inhomogeneities and $1 / T_{2} *$ will be $\sim 2.5$ times smaller compared to $7 \mathrm{~T}$.

In the third study, sequence parameters optimized for EPI-based imaging in OFC were chosen. In order to minimize signal loss due to intra-voxel dephasing and image distortion in the presence of large $B_{0}$-inhomogeneities, high spatial resolution and a coronal slice orientation were employed. One coronal slice out of 16 in the center of the imaged brain region is displayed in Figure 5. All images were corrected for variation of the signal intensity related to the excitation and receive profiles of the RF-coil, which can be significant at $7 \mathrm{~T}$. The GE- and SE-EPI images show the expected $T_{2}{ }^{*}$ - and $T_{2}$-contrast. The short shot-to-shot repetition time in RASER introduces an additional $T_{1}$-weighting in the image contrast, which is opposite to inherent $T_{2}$-contrast. Due to the strong $B_{1}$-inhomogeneity at $7 \mathrm{~T}$ T1-weighting varies significantly across the image volume. Therefore, the cerebrospinal fluid appears to be less bright with RASER compared to GE-EPI and SE-EPI in particular in the center of the coronal slice. Sufficient image SNR in all EPI and RASER time series was present to be able to detect the BOLD response in the OFC. In the EPI images, the image distortions are minor compared to the anatomical $T_{1}$-weighted gradient-echo image.

Figure 6 displays the obtained activation maps. The $t$-score threshold is set so that the same number of activated voxels for each 3D activation map is displayed. The reference number of activated voxels was obtained from the activation map generated from the RASER time series. This approach permits a qualitative assessment of the ratio between 'truly' activated voxels 
and false positives due to spatiotemporally correlated signal instabilities mostly originating from physiological noise. In Figure 6, both EPI methods do not show significant activation above the $t$-score threshold in brain regions listed in table 2 . However, many "activated" voxels in the EPI images are found at the tissue/bone interface and in CSF containing voxels. These regions are known to exhibit high levels of signal instabilities, which often generate false positives. As a result, such activation maps are frequently considered to be unreliable. In Figure 6 , significant activation in RASER data set is found in regions, which are consistent with activation in Figure 4, such as the right superior frontal sulcus (intersecting green lines).

\subsection{Imaging with RASER in the Visual cortex}

RASER can also acquire fMRI data in areas that are easily and equally accessible to EPI-based techniques, such as the visual cortex. Activation studies in these areas, therefore, provide the opportunity to compare the different methods quantitatively.

Figure 5 illustrates images acquired with visual stimulation using RASER, SE-EPI, and GEEPI based fMRI on a single subject. The background images represent the temporal average of the corresponding fMRI time series. Residual ghosting as a result of temporal phase instabilities is seen in the EPI images. In RASER, the time-encoded dimension is not Fouriertransformed; hence, Nyquist ghosting does not occur (Chamberlain et al., 2007). Correcting for shifts of even and odd echoes is therefore obsolete. The linear phase correction of echoes, which is necessary in RASER, solely depends on the imaging parameters, but not on magnetic field inhomogeneities as in EPI.

3.2.1 t-score thresholds of activation maps-The $t$-score statistics considers only the standard deviation of the noise level, but not any spatiotemporal correlations in the noise. As result, at a given $t$-score threshold more false positives are generally observed than predicted by theoretical $t$-score distribution. The general approach is to increase the $t$-score threshold assuming that the most significant voxels correspond to BOLD signal changes, which correlate better with the paradigm than any spatiotemporally correlated noise sources. This very pragmatic approach is often adopted because the contribution of physiological noise varies with the imaging methods and parameters and the current brain physiology of the subjects. Hence, it is virtually impossible to model the noise contribution with sufficient accuracy to correct the $t$-score distribution. As a result, it has been challenging to compare t-maps obtained with different imaging methods. Since various imaging methods exhibit different levels of noise with spatiotemporal correlations, the same $t$-score threshold would not consider the different levels of false positives related to spatiotemporally correlated noise, which is not captured by the standard $t$-score statistics. In this paper, we therefore adopt the approach to set the $t$-score threshold for each imaging method in order to display the same total number of activated voxels.

In particular, the contribution of larger vessel signal changes in the EPI methods result in a higher $t$-score threshold. A lower threshold in EPI would generate $t$-maps with a larger number of activated voxels representing lower, but still significant stimulus-induced signal change as well as artificial temporal correlation of physiological (colored) noise with the stimulus, as indicated by a significant increase in the number of false positives in white matter and regions with signal contribution from cerebrospinal fluid (e.g., in Figure 6). As a consequence, the threshold criteria to determine activated voxels has to be higher for both EPI methods compared to RASER. These assumptions will be discussed in more detail later in the section "contrastto-noise analysis".

In Figure 7, thresholded $t$-scores representing statistical significance of the mean difference between the on and the off-conditions of the visual stimulation paradigm are superimposed on the images. The thresholds were highest for GE-EPI followed by SE-EPI and then RASER. In 
all subjects, significant and similar activation in the primary visual cortex (V1) was found in the maps obtained with the three sequences.

3.2.2 Contrast-to-noise analysis-Figure 8 illustrates the trial averages, that is, the mean of on and off periods of stimulus of the averaged time courses, based in the same ROIs in the primary visual cortex used in the statistical noise analysis. GE-EPI has the largest maximum relative signal change $\Delta \mathrm{S} / \mathrm{S}$ (Table 3 ), as expected, because all deoxyhemoglobin containing vessels, irrespective of size, contribute. Functional signal changes seen in SE-EPI are less because contributions from blood vessels with diameters of $\sim 10 \mu \mathrm{m}$ or larger decrease significantly (Fujita, 2001). The maximal relative signal change of RASER is the smallest, as expected, since it is purely $T_{2}$ weighted without any residual $T_{2} *$ contribution as in SE-EPI.

RASER is inherently less sensitive and yields images with poorer intrinsic SNR compared to phase encoded $\boldsymbol{k}$-space acquisition such as EPI (Chamberlain and Garwood, 2009). The SNR of a single image (determined by "thermal noise") was $200 \pm 80,90 \pm 30,19 \pm 7$ for GE-EPI, SEEPI and RASER (the error represents the standard deviation of the variability between subjects). In all cases the $T_{R}$ was $2 \mathrm{~s}$ and the excitation pulse was calibrated to generate the same flip angle. The difference between the SE and GE-EPI images is likely to arise from the use of a surface coil, where excitation and refocusing pulse angles greater or less than $90^{\circ}$ and $180^{\circ}$, respectively, lead to SNR loss with SE sequences. Signal fluctuations in an fMRI time series, however, contain physiological contributions that can dominate over thermal noise. These contributions arise mainly from heart beat and respiration, as well as fluctuations that have a neuronal basis and are considered as "noise" for stimulus- or task-evoked functional activation (Biswal et al., 1996, Kruger and Glover, 2001, Purdon and Weisskoff, 1998).

For the noise analysis, the same reference ROI in the activated primary visual cortex for the three imaging techniques for each subject (for details see Methods, Analysis of the functional time-series) was used. The average number of voxels in the reference ROIs was $180 \pm 80$, where the error represents the standard deviation across subjects. The noise analysis was performed on two different kinds of data: first, all complex-valued time courses in the selected ROI were averaged and the temporal noise analysis was performed afterwards on the spatially averaged time course. This is referred to as the 'average across ROI' analysis in Table 3. In the second approach, the temporal noise analysis was performed in each voxel time course individually prior to averaging the noise measures across the ROI ('voxel-by-voxel' analysis in Table 3). It is assumed that physiological noise, which is dominated by cardiac activity and breathing, is largely spatially correlated; it has a component, likely mediated by capillaries, that is not correlated on the spatial scale of MRI voxels and is detected in SE time series (Yacoub et al., 2005). Instrumental instabilities can also contribute to temporal fluctuations and would be spatially correlated. Thermal noise is statistically independent between different voxels. Hence, if time courses are averaged over the different voxels before the analysis, the contribution of thermal noise and spatially incoherent fluctuations is reduced, while the spatially coherent noise persists.

The ratio of standard deviation of the physiological and the intrinsic thermal noise, $\sigma_{\text {phys }}$ ' $\sigma_{\text {intr }}$, of a ROI in the visual cortex is listed in Table 3 for both the 'average across ROI' and the 'voxel-by-voxel' analysis. The imaging parameters were selected to provide the same contribution of thermal noise in all imaging methods.

For the 'voxel-by-voxel' analysis, $\sigma_{\text {phys }} / \sigma_{\text {intr }}$ is larger than unity in all cases, indicating the presence of significant physiological contributions in all cases, including RASER. However, $\sigma_{\text {phys }} / \sigma_{\text {intr }}$ is smaller by a factor of five in RASER compared to SE-EPI, and is largest for GEEPI. Despite the significantly different intrinsic SNR of the three methods, the "temporal" SNR (tSNR), calculated as the signal amplitude versus the standard deviation of the temporal 
fluctuations, is the same for all three methods. The success of fMRI studies is determined by tSNR rather than intrinsic SNR, and in this respect, RASER does as well as SE-EPI and GEEPI. Functional contrast-to-noise ratio (CNR) is calculated as the ratio of the stimulus induced signal change, $\Delta S$, divided by the standard deviation of the temporal noise; hence, it is equal to $\triangle \mathrm{S} \times \mathrm{tSNR}$. CNR for RASER is lowest among the three different methods, despite the equality in $\mathrm{tSNR}$. This is ascribed to the larger $\Delta \mathrm{S}$ of the SE-EPI and especially for the GE-EPI (Fig.5) due to increasing but often undesirable contributions from large veins. The mean $t$-score is proportional to the CNR as expected (Table 3).

In case of the 'average across ROI' analysis, differences in $\sigma_{\text {phys }} / \sigma_{\text {intr }}$ among the three approaches increase dramatically; RASER improves compared SE-EPI (i.e. has a lower value for $\sigma_{\text {phys }}\left(\sigma_{\text {intr }}\right)$, which in turn improves relative to GE-EPI. These findings indicate that in RASER, and to a lesser extend in SE-EPI, thermal noise and spatially incoherent physiological noise dominate temporal fluctuations of individual voxel time courses. In contrast, spatially coherent fluctuations still contributed to SE-EPI and dominate GE-EPI time series, rendering spatial averaging ineffective. Thus, tSNR becomes lower for GE-EPI vs. SE-EPI with spatial averaging as previously demonstrated (Yacoub et al., 2005) and improves for RASER compared to both SE-EPI and GE-EPI.

\section{Discussion}

The inability to detect MR signals around air filled cavities due to the deleterious effects of magnetic field inhomogeneities has prevented fMRI from being a truly "whole brain" functional imaging method. Significant regions of the brain, in particular large sections of the frontal and temporal lobes, have remained inaccessible to the technique that has evolved into the most commonly used method for studying human brain function. Substantial efforts have been undertaken to minimize this signal loss, ranging from implementing compensation gradients in the pulse sequence to reducing the voxel size (Cordes et al., 2000, Cusack et al., 2005, De Panfilis and Schwarzbauer, 2005, de Zwart et al., 2006, Deichmann and Turner, 2001, Deichmann et al., 2002, Du et al., 2007, Glover, 1999, Golay et al., 2004, Gu et al., 2002, Heberlein and Hu, 2004, Juchem et al., 2006, Koch et al., 2006, Liu and Ogawa, 2006, Preibisch et al., 2003, Schmidt et al., 2005, Takahashi et al., 2001, Weiskopf et al., 2006, Weiskopf et al., 2007, Wilson et al., 2003, Wilson and Jezzard, 2003, Yang et al., 2004, Yang et al., 2006). None of these previous efforts have fully resolved this problem. RASER overcomes this disadvantage. Although comparisons presented in this paper were limited to EPI, similar advantages would be applicable as well with respect to the SPIRAL acquisition strategy, which is also used in fMRI studies, albeit to a lesser extend. Since $T_{2}{ }^{*}$-effects do not contribute at all to the signal in RASER, intra-voxel dephasing and, hence, signal losses in brain regions with large susceptibility-induced background gradients, are eliminated.

The insensitivity of RASER to $B_{0}$ inhomogeneities occurs for two reasons. First, RASER is purely $T_{2}$-weighted, without any residual $T_{2}{ }^{*}$-component as in SE-EPI. The GE-EPI images with moderate spatial resolution show significant signal loss and geometric distortion at the frontal pole. SE-EPI images exhibit less signal loss, since the $T_{2} *$-weighting is determined by half of the duration of the echo train $(0.5 \cdot 32 \mathrm{~ms})$, while the $T_{2} *$-weighting of the echoes in GEEPI varies between $T_{E^{-}} 0.5 \cdot 32 \mathrm{~ms}$ and $T_{E}+0.5 \cdot 32 \mathrm{~ms}$, where the echo time, $T_{E}$, was $25 \mathrm{~ms}$.

Second, the phase-encoding blips of EPI are replaced by time-encoding gradients, which have similar amplitude as the readout gradient; this is approximately 30 times larger than the dephase gradient for phase-encoding in EPI. The phase-encoding of the third dimension in RASER is not affected by constant background gradients since it manifests itself as a constant phase offset in this dimension in the image domain. Hence, RASER images exhibit neither signal loss nor distortions in the presence of magnetic field inhomogeneities. 
Intra-voxel dephasing in the EPI image acquisition scheme is determined by the duration of the echo train. This duration can be shortened by reducing the number of acquired phaseencoding steps, which can be achieved in two ways: The FOV can be reduced, while maintaining spatial resolution, for example, by using parallel imaging and/or a slab-selective refocusing pulse in the phase-encoded dimension. The other possibility is to split phaseencoding into multiple acquisitions and combine the segments prior to image reconstruction. This technique can reduce $T_{2}{ }^{*}$-contribution significantly at the expense of lower temporal resolution and higher sensitivity to physiological noise. None of these approaches eliminates this problem completely. RASER, however, is inherently insensitive to this effect.

When magnetic field inhomogeneities are not large enough to render MR signals completely undetectable, but sufficiently large compared to spatial encoding gradients in the image acquisition, they give rise to another problem: image distortion and blurring in EPI and SPIRAL images, respectively. With EPI, these distortions preclude accurate registrations of functional data with anatomical images. In SPIRAL images, they degenerate the accuracy of the functional maps. Often, but not always, these distortions are corrected in a post-processing step using a $B_{0}$ - field map which is usually acquired separately. In RASER, phase-encoding is replaced with time-encoding, which uses a much larger gradient. Hence, RASER is not susceptible to distortions even with increased susceptibility induced background gradients at ultra-high magnetic field strength.

As an alternative approach, z-shim methods may alleviate signal loss and image distortions (Du et al., 2007, Glover, 1999, Gu et al., 2002, Heberlein and Hu, 2004, Takahashi et al., 2001). However, even in the favorable situation - as shown with the coronal images with high spatial resolution - when the signal in the OFC is completely retained and image distortions are negligible for EPI-based techniques, RASER exhibits a superior detection sensitivity of the BOLD signal changes compared to EPI methods. The reason is that RASER is inherently less susceptible to temporal variation of $B_{0}$. Z-shimming does not reduce signal fluctuations related to such instabilities.

A prominent source of noise in fMRI time series is due to physiological processes unrelated to brain activity, such as breathing and cardiac pulsation; as such, they are deleterious to brain function studies. Such physiologically induced temporal signal fluctuations are expected to be minimized in true $T_{2}$-weighted signal; accordingly, physiological sources contribute less to the temporal signal instabilities in RASER compared to EPI acquisition schemes. This is attributed to the inherent insensitivity of RASER to inhomogeneities of the magnetic field.

Recent research efforts in fMRI move towards ultrahigh magnetic fields mainly for two reasons. First, the intrinsic SNR increases proportional to magnetic field strength (Ugurbil et al., 2006). Second, even more prominent gains are observed in the CNR in fMRI experiments, especially for signals of microvascular origin that are more accurately localized relative to the source of neuronal activity (Ugurbil et al., 2006). Both effects are advantageous for high resolution fMRI studies. In particular, $T_{2}$-functional contrast at ultrahigh fields is expected to be more confined to the site of neuronal activity than the signals detected with $T_{2}{ }^{*}$-contrast in GE-EPI(Fujita, 2001). RASER is a purely $T_{2}$-weighted sequence without residual $T_{2}{ }^{*}$-effects that are present even in SE-EPI. Therefore, RASER is a promising sequence for high specificity fMRI at ultrahigh magnetic fields.

Despite the fact that the $T_{2}$-weighting in RASER produces signal changes with a smaller amplitude than $T_{2}{ }^{*}$-weighted sequences, experimental results demonstrate that RASER has excellent sensitivity to neuronal activation due to the significant reduction of physiological noise. RASER gains are particularly pronounced for short $T_{2} *$ regions, where EPI-based methods fail to detect a functional response. 
An apparent limitation of the current version of RASER is its high power deposition as a result of an excitation pulse with high bandwidth and two high flip-angle refocusing pulses per acquired image in combination with short repetition times for sufficient temporal resolution in fMRI experiments. This is a general problem for $T_{2}$-weighted fast imaging sequences. The more commonly used $T_{2}$-weighted SE-EPI sequence appears advantageous in this respect compared to RASER since it requires only one refocusing pulse. However, to overcome problems resulting from $T_{2}{ }^{*}$-contribution, SE-EPI is usually segmented, increasing the power deposition per image. Another $T_{2}$-weighted method is HASTE (Poser and Norris, 2007).

HASTE requires high flip angle refocusing pulses for each $\boldsymbol{k}$-space line. To reduce the length of the echo train, partial Fourier acquisition and parallel imaging must be used. In contrast, the spatial resolution in RASER can be tuned by adjusting the bandwidth-time product of the chirp pulse. Furthermore, as RASER has only two refocusing pulses it is more power efficient than HASTE.

In conclusion, RASER is a novel and unique technique for fMRI applications. It drastically improves the applicability and precision of fMRI experiments. Further technical developments include the implementation of parallel imaging techniques to improve brain coverage at acceptable spatial and temporal resolution, while reducing the power deposition per acquired volume.

\section{Acknowledgments}

The authors are grateful to Drs Edward Auerbach, Gregor Adriany, Peter Anderson, and John Strupp for technical support and Dr Cheryl Olman for help with the image registration and analysis package FSL. The authors also thank Dr. Ryan Chamberlain, Tram Nguyen (Max-Planck-Institute for Biological Cybernetics, Tuebingen, Germany) and Noam Ben-Eliezer (Weizmann Institute, Rehovot, Israel) for helpful discussions. Financial support from NIH grants P41 RR008079 and P30 NS057091, the Keck Foundation, and the Mind Institute is gratefully acknowledged.

\section{References}

Bellgowan PSF, Bandettini PA, van Gelderen P, Martin A, Bodurka J. Improved BOLD detection in the medial temporal region using parallel imaging and voxel volume reduction. Neuroimage 2006;29:1244-1251. [PubMed: 16242347]

Birn R, Bandettini PA. The Effect of T2' Changes on Spin-Echo EPI derived Brain Activation Maps. Proc Int Soc Magn Reson Med Sci Meet Exhib 2002;10:1324.

Biswal B, DeYoe EA, Hyde JS. Reduction of physiological fluctuations in fMRI using digital filters. Magn Res Med 1996;35:107-113.

Bosch V. Statistical analysis of multi-subject fMRI data: Assessment of focal activations. J Magn Reson Imaging 2000;11:61-64. [PubMed: 10676622]

Bruder H, Fischer H, Reinfelder H-E, Schmitt F. Image Reconstruction for Echo Planar Imaging with Nonequidistant k-Space Sampling. Magn Res Med 1992;23:311-323.

Chamberlain R, Park JY, Corum C, Yacoub E, Ugurbil K, Jack CR, Garwood M. RASER: A new ultrafast magnetic resonance Imaging method. Magn Res Med 2007;58:794-799.

Chamberlain R, Garwood M. General time-encoding description and improved RASER imaging. Proc Int Soc Magn Reson Med Sci Meet Exhib 2009;17:2671.

Chen NK, Wyrwicz AM. Optimized distortion correction technique for echo planar imaging. Magn Res Med 2001;45:525-528.

Constable RT, Spencer DD. Composite image formation in z-shimmed functional MR imaging. Magn Res Med 1999;42:110-117.

Cordes D, Turski PA, Sorenson JA. Compensation of susceptibility-induced signal loss in echo-planar imaging for functional applications. Magn Reson Imaging 2000;18:1055-1068. [PubMed: 11118760] 
Cusack R, Russell B, Cox SML, De Panfilis C, Schwarzbauer C, Ansorge R. An evaluation of the use of passive shimming to improve frontal sensitivity in fMRI. Neuroimage 2005;24:82-91. [PubMed: 15588599]

De Panfilis C, Schwarzbauer C. Positive or negative blips? The effect of phase encoding scheme on susceptibility-induced signal losses in EPI. Neuroimage 2005;25:112-121. [PubMed: 15734348]

de Zwart JA, van Gelderen P, Golay X, Ikonomidou VN, Duyn JH. Accelerated parallel imaging for functional imaging of the human brain. NMR Biomed 2006;19:342-351. [PubMed: 16705634]

Deichmann R, Turner R. Compensation of susceptibility induced BOLD sensitivity losses in Echo-Planar fMRI imaging. Neuroimage 2001;13:S8-S8.

Deichmann R, Josephs O, Hutton C, Corfield DR, Turner R. Compensation of susceptibility-induced BOLD sensitivity losses in echo-planar fMRI Imaging. Neuroimage 2002;15:120-135. [PubMed: 11771980]

Deichmann R, Gottfried JA, Hutton C, Turner R. Optimized EPI for fMRI studies of the orbitofrontal cortex. Neuroimage 2003;19:430-441. [PubMed: 12814592]

Du YPP, Dalwani M, Wylie K, Claus E, Tregellas JR. Reducing susceptibility artifacts in fMRI using volume-selective z-shim compensation. Magn Res Med 2007;57:396-404.

Fujita N. Extravascular contribution of blood oxygenation level-dependent signal changes: A numerical analysis based on a vascular network model. Magn Res Med 2001;46:723-734.

Glover GH. 3D z-shim method for reduction of susceptibility effects in BOLD fMRI. Magn Res Med 1999;42:290-299.

Golay X, de Zwart JA, Ho Y-CL, Sitoh Y-Y. Parallel imaging techniques in functional MRI. Top Magn Reson Imaging 2004;15:255-65. [PubMed: 15548956]

Griswold MA, Jakob PM, Heidemann RM, Nittka M, Jellus V, Wang JM, Kiefer B, Haase A. Generalized Autocalibrating Partially Parallel Acquisitions (GRAPPA). Magn Res Med 2002;47:1202-1210.

Gu H, Feng HH, Zhan W, Xu S, Silbersweig DA, Stern E, Yang YH. Single-shot interleaved Z-shim EPI with optimized compensation for signal losses due to susceptibility-induced field inhomogeneity at 3 T. Neuroimage 2002;17:1358-1364. [PubMed: 12414275]

Heberlein KA, Hu XP. Simultaneous acquisition of gradient-echo and asymmetric spin-echo for singleshot z-shim: Z-SAGA. Magn Res Med 2004;51:212-216.

Juchem C, Muller-Bierl B, Schick F, Logothetis NK, Pfeuffer J. Combined passive and active shimming for in vivo MR spectroscopy at high magnetic fields. J Magn Reson 2006;183:278-289. [PubMed: 17011219]

Koch KM, Brown PB, Rothman DL, de Graaf RA. Sample-specific diamagnetic and paramagnetic passive shimming. J Magn Reson 2006;182:66-74. [PubMed: 16814580]

Kruger G, Glover GH. Physiological noise in oxygenation-sensitive magnetic resonance imaging. Magn Res Med 2001;46:631-637.

Lin FH, Huang TY, Chen NK, Wang FN, Stufflebeam SM, Belliveau JW, Wald LL, Kwong KK. Functional MRI using regularized parallel imaging acquisition. Magn Res Med 2005;54:343-353.

Liu GX, Ogawa S. EPI image reconstruction with correction of distortion and signal losses. J Magn Reson Imaging 2006;24:683-689. [PubMed: 16892198]

Moeller S, Van de Moortele PF, Goerke U, Adriany G, Ugurbil K. Application of parallel imaging to fMRI at 7 Tesla utilizing a high 1D reduction factor. Magn Res Med 2006;56:118-129.

Norris DG, Zysset S, Mildner T, Wiggins CJ. An investigation of the value of spin-echo-based fMRI using a Stroop color-word matching task and EPI at 3 T. Neuroimage 2002;15:719-726. [PubMed: 11848715]

Ojemann JG, Akbudak E, Snyder AZ, McKinstry RC, Raichle ME, Conturo TE. Anatomic localization and quantitative analysis of gradient refocused echo-planar fMRI susceptibility artifacts. Neuroimage 1997;6:156-167. [PubMed: 9344820]

Park JY, Garwood M. Spin-Echo MRI Using pi/2 and pi Hyperbolic Secant Pulses. Magn Res Med 2009;61:175-187.

Poser BA, Versluis MJ, Hoogduin JM, Norris DG. BOLD contrast sensitivity enhancement and artifact reduction with multiecho EPI: Parallel-acquired inhomogeneity-desensitized fMRI. Magn Res Med 2006;55:1227-1235. 
Poser BA, Norris DG. Fast spin echo sequences for BOLD functional MRI. MAGMA 2007;20:11-17. [PubMed: 17245581]

Preibisch C, Pilatus U, Bunke R, Hoogenraad F, Zanella F, Lanfermann H. Functional MRI using sensitivity-encoded echo planar imaging (SENSE-EPI). Neuroimage 2003;19:412-421. [PubMed: 12814590]

Pruessmann KP, Weiger M, Scheidegger MB, Boesiger P. SENSE: Sensitivity encoding for fast MRI. Magn Res Med 1999;42:952-962.

Purdon PL, Weisskoff RM. Effect of temporal autocorrelation due to physiological noise and stimulus paradigm on voxel-level false-positive rates in fMRI. Hum. Brain Mapp 1998;6:239-249.

Schmidt CF, Degonda N, Luechinger R, Henke K, Boesiger P. Sensitivity-encoded (SENSE) echo planar fMRI at 3T in the medial temporal lobe. Neuroimage 2005;25:625-641. [PubMed: 15784442]

Strupp JP. Stimulate: A GUI based fMRI analysis software package. Neuroimage 1996;3:S607.

Takahashi T, Xiao RT, Kawano K, Iijima T. Assessment of the practicability of z-Shim method in fMRI. Neuroimage 2001;13:S47-S47.

Tal A, Frydman L. Spatial encoding and the single-scan acquisition of high definition MR images in inhomogeneous fields. J Magn Reson 2006;182:179-194. [PubMed: 16843690]

Treisman AM, Fearnley S. The Stroop test: Selective attention to colours and words. Nature 1969;222:437-439. [PubMed: 5768618]

Ugurbil K, Hu XP, Chen W, Zhu XH, Kim SG, Georgopoulos A. Functional mapping in the human brain using high magnetic fields (vol B 354, pg 1195, 1999). Philos Trans R Soc Lond B Biol Sci 1999;354:2084-2084.

Ugurbil K, Toth L, Kim DS. How accurate is magnetic resonance imaging of brain function? Trends Neurosci 2003;26:108-114. [PubMed: 12536134]

Ugurbil, K.; Chen, W.; Harel, N.; van de Moortele, PF.; Yacoub, E.; Zhu, X.; Uludag, K. Wiley Encyclopedia of Biomedical Engineering. Akay, M., editor. John Wiley \& Sons; Hoboken: 2006. p. 647-668.

Weiskopf N, Hutton C, Josephs O, Deichmann R. Optimal EPI parameters for reduction of susceptibilityinduced BOLD sensitivity losses: A whole-brain analysis at 3 T and 1.5 T. Neuroimage 2006;33:493504. [PubMed: 16959495]

Weiskopf N, Hutton C, Josephs O, Turner R, Deichmann R. Optimized EPI for fMRI studies of the orbitofrontal cortex: compensation of susceptibility-induced gradients in the readout direction. MAGMA 2007;20:39-49. [PubMed: 17268781]

Wilson JL, Jenkinson M, Jezzard P. Protocol to determine the optimal intraoral passive shim for minimisation of susceptibility artifact in human inferior frontal cortex. Neuroimage 2003;19:18021811. [PubMed: 12948734]

Wilson JL, Jezzard P. Utilization of an intra-oral diamagnetic passive shim in functional MRI of the inferior frontal cortex. Magn Res Med 2003;50:1089-1094.

Yacoub E, Van De Moortele PF, Shmuel A, Ugurbil K. Signal and noise characteristics of Hahn SE and GE BOLD fMRI at $7 \mathrm{~T}$ in humans. Neuroimage 2005;24:738-750. [PubMed: 15652309]

Yacoub E, Shmuel A, Logothetis N, Ugurbil K. Robust detection of ocular dominance columns in humans using Hahn Spin Echo BOLD functional MRI at 7 Tesla. Neuroimage 2007;37:1161-1177. [PubMed: 17702606]

Yacoub E, Harel N, Ugurbil K. High-field fMRI unveils orientation columns in humans. Proc Natl Acad Sci U S A 2008;105:10607-10612. [PubMed: 18641121]

Yang QX, Wang JL, Smith MB, Meadowcroft M, Sun XY, Eslinger PJ, Golay X. Reduction of magnetic field inhomogeneity artifacts in echo planar imaging with SENSE and GESEPI at high field. Magn Res Med 2004;52:1418-1423.

Yang QX, Mao WH, Wang JH, Smith MB, Lei H, Zhang XL, Ugurbil K, Chen W. Manipulation of image intensity distribution at 7.0 T: Passive RF shimming and focusing with dielectric materials. J Magn Reson Imaging 2006;24:197-202. [PubMed: 16755543]

Zysset S, Muller K, Lohmann G, von Cramon DY. Color-word matching stroop task: Separating interference and response conflict. Neuroimage 2001;13:29-36. [PubMed: 11133306] 


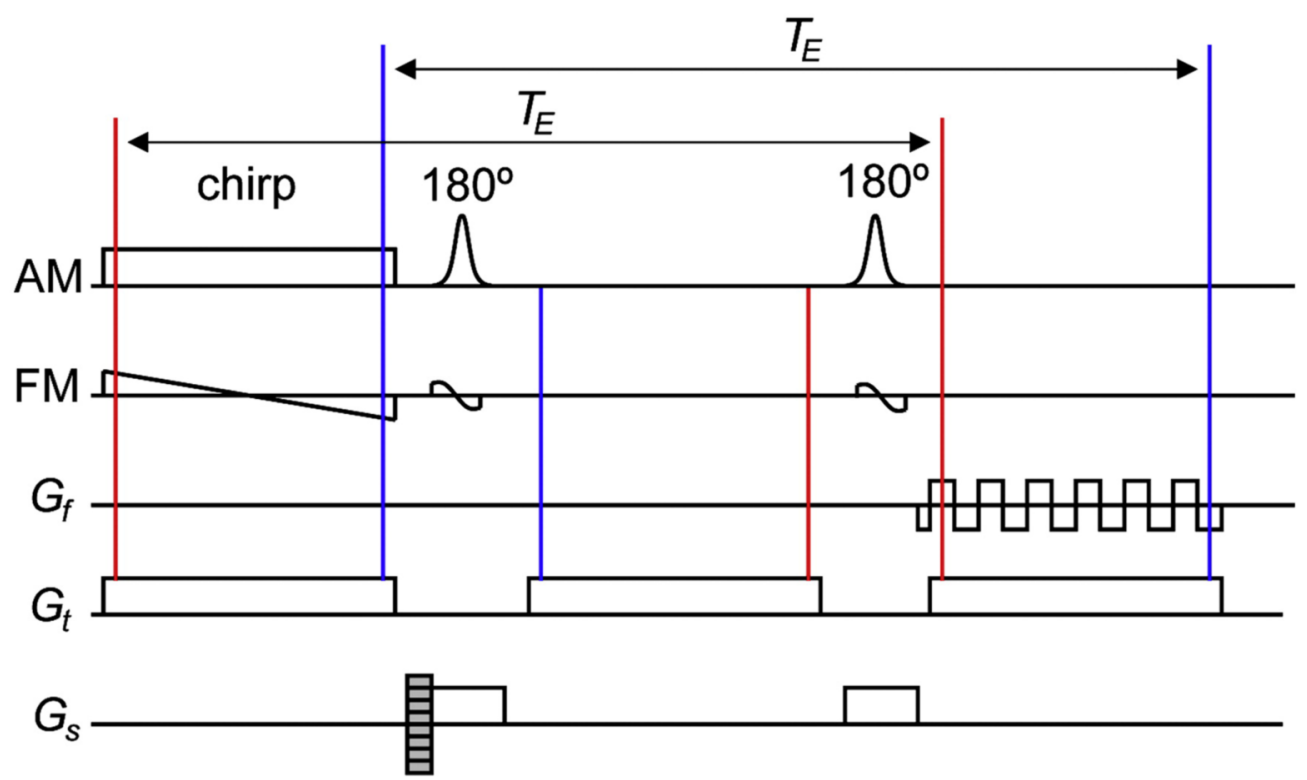

Figure 1.

2D single-shot RASER pulse sequence (without gray-shaded gradient pulses). The gray shaded gradient pulses for phase-encoding of the third spatial dimension are added if the sequence is used in a multi-shot multi-slice variant. 

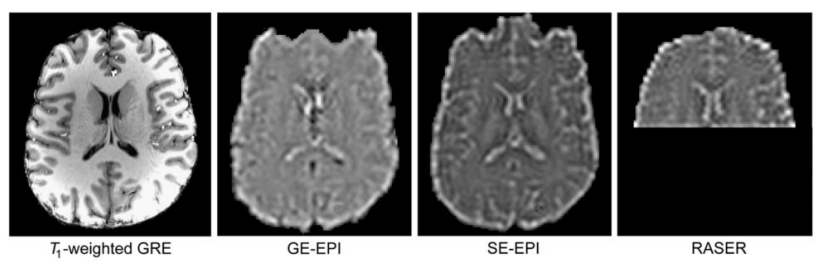

Figure 2.

A $T_{1}$-weighted anatomical image and a corresponding single slice from axial multi-slice GEEPI, SE-EPI, and RASER data sets of a single subject. The average image of the respective time series shows the signal loss and geometric distortion generated by the EPI-based methods in the orbitofrontal cortex. In RASER, only half of the FOV is acquired in the time-encoded dimension. Signal variations due to inhomogeneities of the radiofrequency fields are removed from all images. 

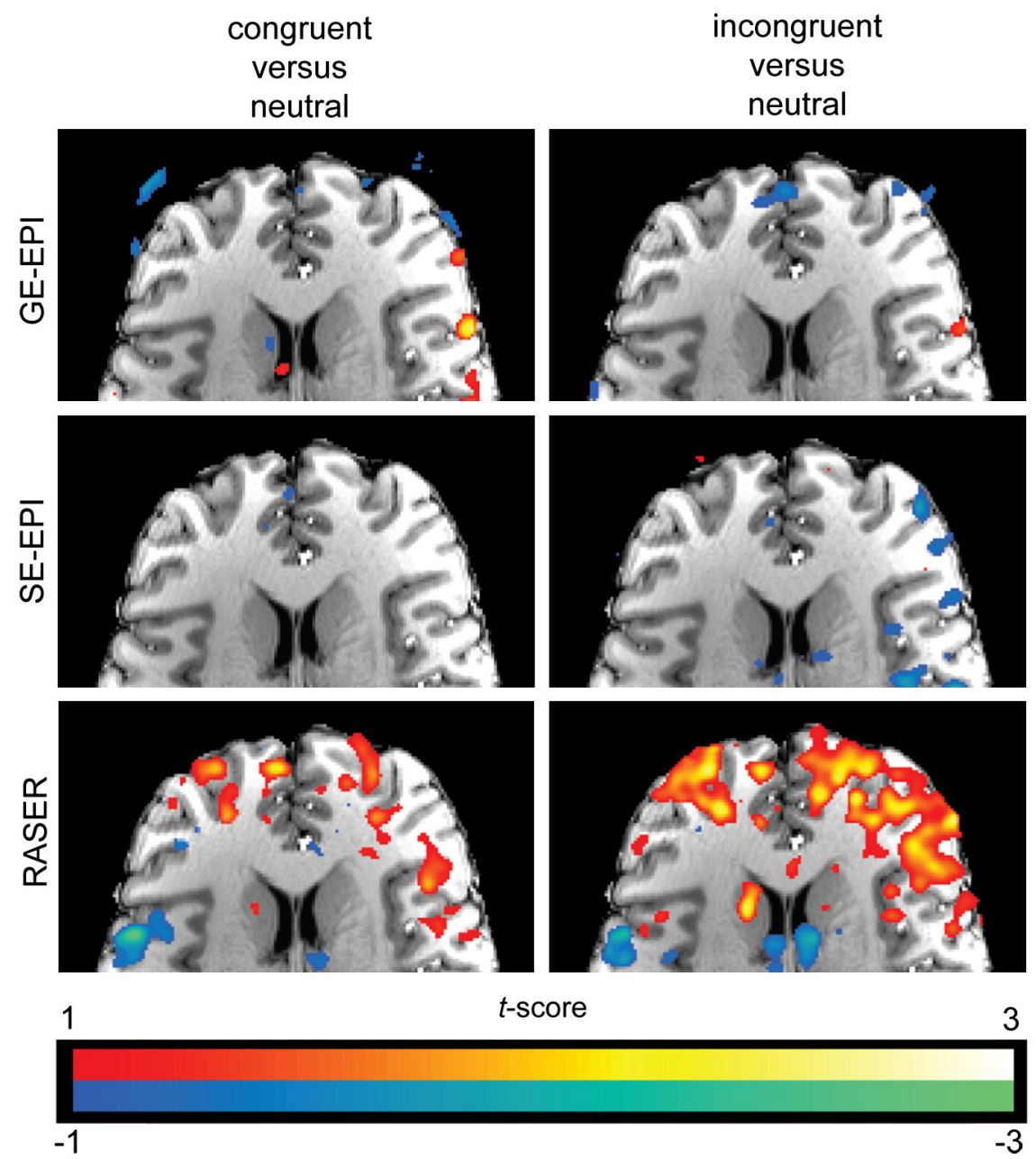

Figure 3.

$t$-maps corresponding GE-EPI, SE-EPI and RASER data displayed in Figure 2 The color-code corresponds to congruent versus neutral and incongruent versus neutral condition of the Strooptest. The $t$-maps are superimposed on a $T_{1}$-weighted anatomical scan. 


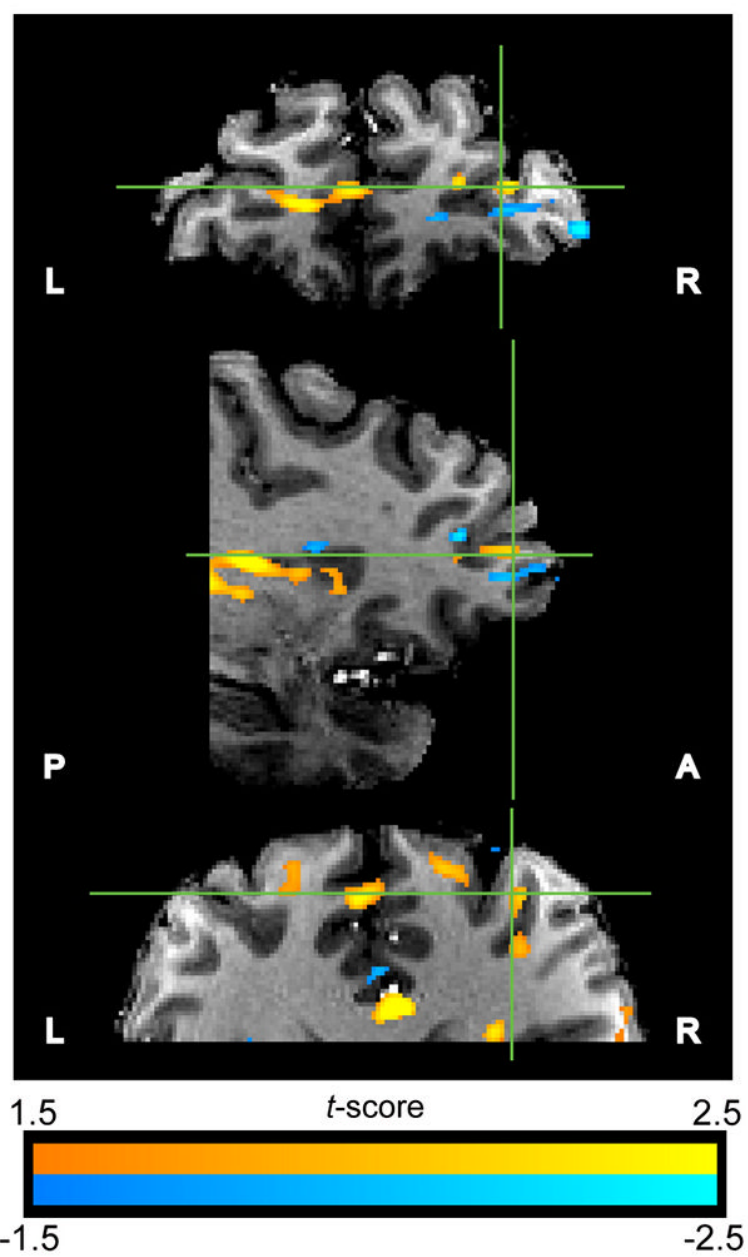

Figure 4.

The $t$-maps obtained from RASER time series superimposed on a $T_{1}$-weighted anatomical scan. The $t$-map represents the normalized average of the $t$-maps of three different sessions of the same subject. Only voxel clusters with more than 125 activated voxels are displayed. The color code represents $t$-scores of incongruent versus congruent condition of the Stroop-task. Activated brain regions are located in the left and right transverse frontopolar gyrus (both positive), the right transverse frontopolar gyrus (negative), the right medial frontal gyrus (negative), the left superior rostral gyrus (positive), and the cingulate cortex (positive). 


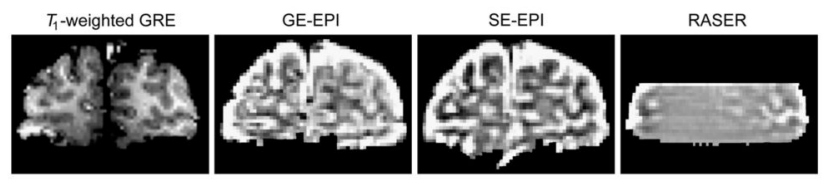

Figure 5.

A coronal slice of an anatomical $T_{1}$-weighed gradient-echo (GRE) data set and of multi slice GE-EPI, SE-EPI and RASER time-averaged data sets of a single subject. The images were acquired with high spatial resolution to minimize signal loss and geometric distortions of EPI images due to large $B_{0}$-inhomogeneities. The EPI images were acquired in four segments, while RASER was zoomed in the time-encoded dimension. Signal variations due to inhomogeneities of the radiofrequency fields are removed from all images. 


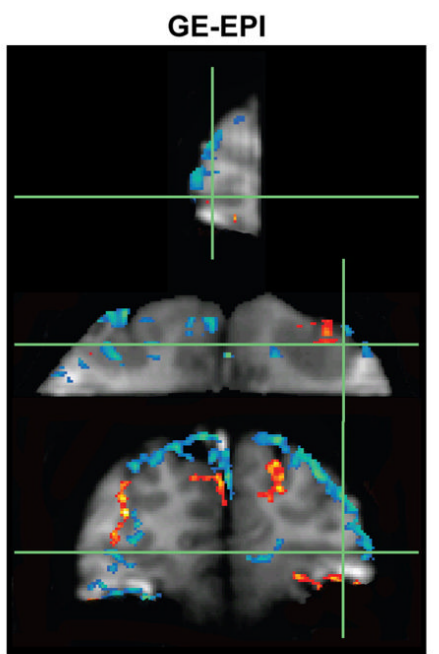

$2.9<|t|<8.9$

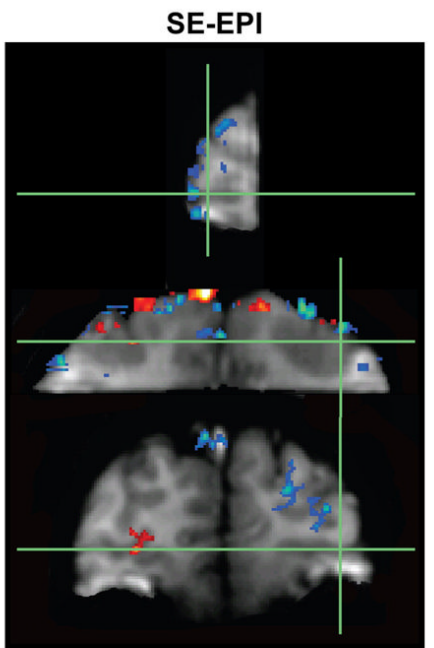

$1.6<|t|<7.6$

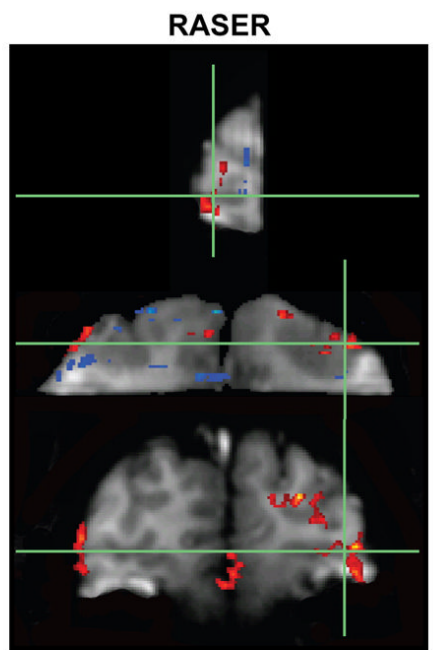

$1.3<|t|<7.3$

Figure 6.

$t$-maps obtained from the corresponding image time series displayed in Figure 5 comparing the sensitivity of GE-EPI, SE-EPI and RASER to detect BOLD signal changes in OFC related to the incongruent versus congruent condition of the Stroop test. The lower $t$-score threshold was determined by selecting the same number of "activated" voxels in the 3D $t$-maps for all imaging methods. The red-to-yellow color scale indicates increasing positive $t$-scores, while the blue-to-green color ranges represents decreasing negative $t$-values. 

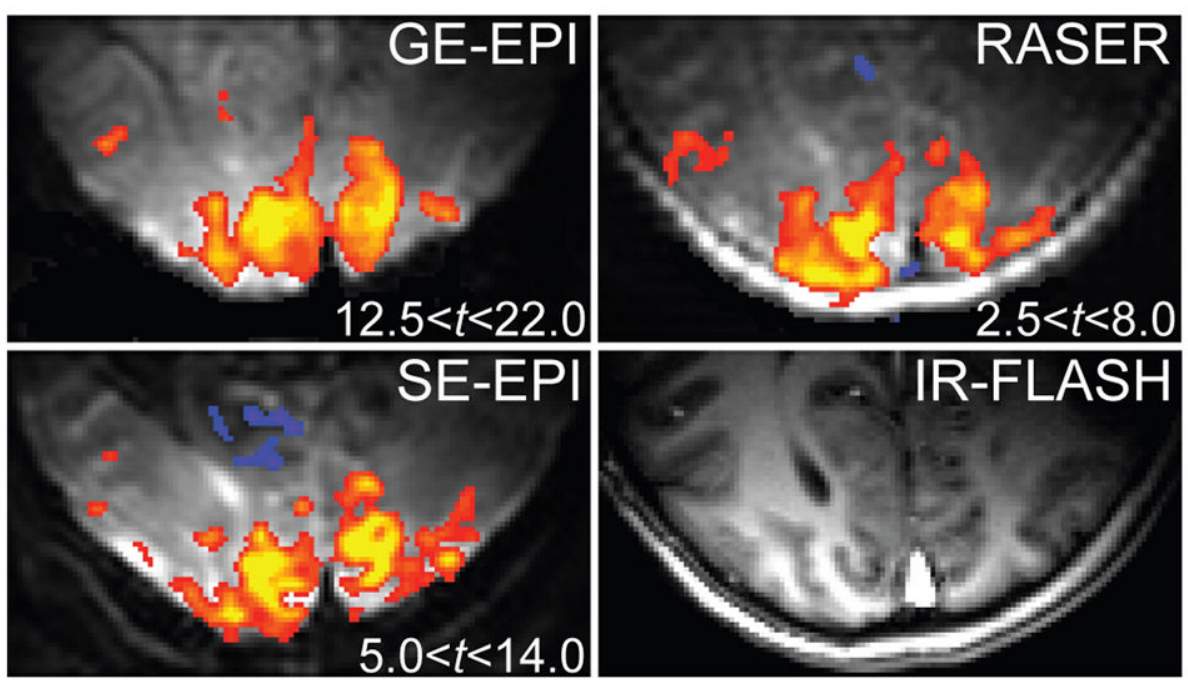

Figure 7.

$t$-maps showing activation in the primary visual cortex related to the visual stimulation paradigm based on the circular flashing checkerboard. The range of $t$-scores correspond the red-to-yellow color scale. For comparison, a $T_{1}$-weighted anatomical scan acquired with IRFLASH is shown. 


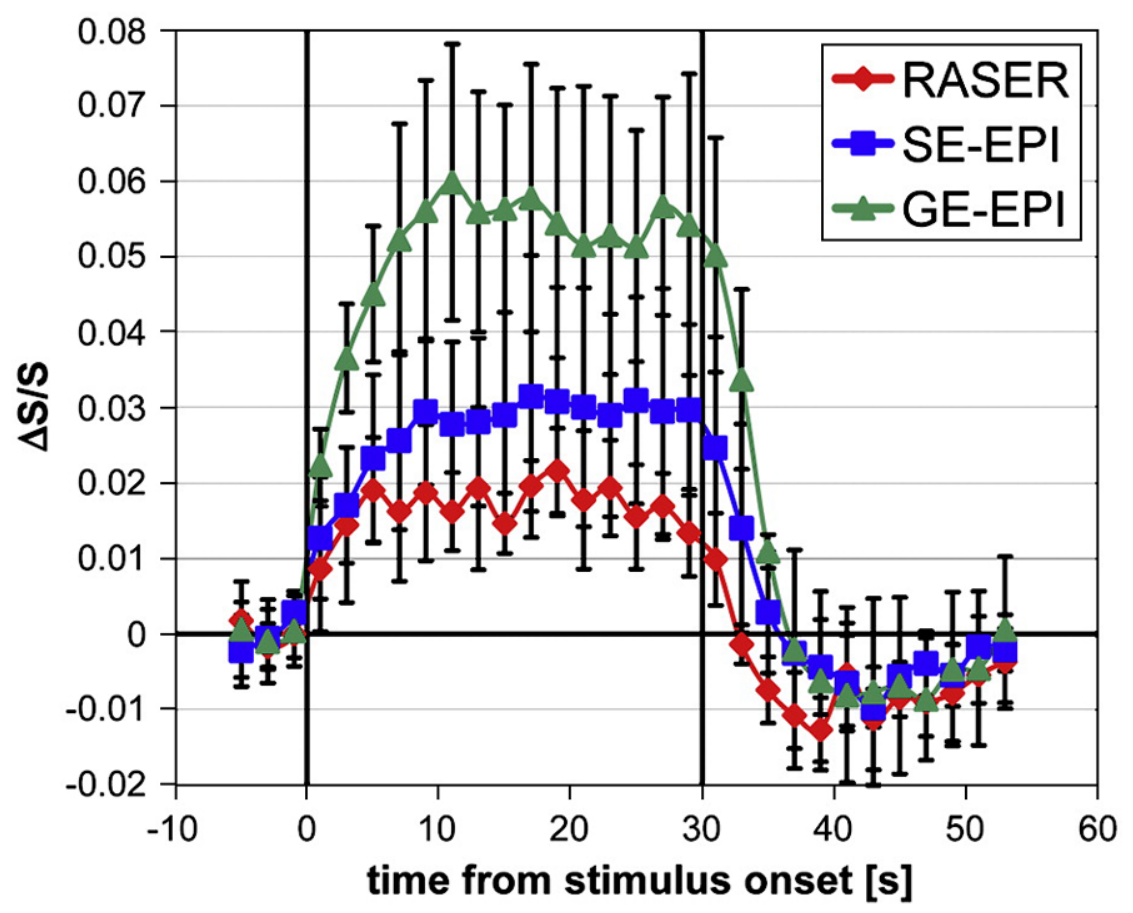

Figure 8.

Trial averages of a ROI including activated voxels in at least one of the imaging techniques averaged over all subjects. The ROIs for "contrast-to-noise analysis" in the primary visual cortex were used to generate the average time courses. The vertical lines at $0 \mathrm{~s}$ and $30 \mathrm{~s}$ mark start and end of the stimulation period. The error bars represent the standard deviation of variations between subjects. 
Table 1

Overview over fMRI experiments

\begin{tabular}{|c|c|c|c|c|}
\hline brain region paradigm & slice orientation & spatial resolution & matrix size & \# of subjects \\
\hline $\begin{array}{l}\text { orbitofrontal cortex } \\
\text { Stroop - incongruent/neutral congruent/neutral }\end{array}$ & axial & $(3 \mathrm{~mm})^{3}$ & $\begin{array}{l}\text { RASER: } 64 \times 32 \times 8 \text { EPI: } \\
\quad 64 \times 64 \times 8\end{array}$ & 3 \\
\hline $\begin{array}{l}\text { orbitofrontal cortex } \\
\text { Stroop - incongruent/congruent }\end{array}$ & axial & $(3 \mathrm{~mm})^{3}$ & $\begin{array}{l}\text { RASER: } 64 \times 32 \times 8 \text { EPI: } \\
\quad 64 \times 64 \times 12\end{array}$ & $\begin{array}{c}2 \text { (3 sessions for } 1 \\
\text { subject) }\end{array}$ \\
\hline $\begin{array}{l}\text { orbitofrontal cortex } \\
\text { Stroop - incongruent/congruent }\end{array}$ & coronal & $1.5 \times 1.5 \times 2 \mathrm{~mm}^{3}$ & $\begin{array}{l}\text { RASER: } 128 \times 24 \times 164- \\
\text { seg. EPI: } 128 \times 128 \times 16\end{array}$ & 2 \\
\hline $\begin{array}{l}\text { primary visual cortex } \\
\text { flashing checkerboard }\end{array}$ & oblique & $2 \times 2 \times 3 \mathrm{~mm}^{3}$ & $64 \times 32 \times 1$ & 5 \\
\hline
\end{tabular}


Table 2

Location of correlated signal changes of incongruent versus congruent condition of the word-color match Stroop test in the frontal lobe based on the $t$-map shown in Figure 4.

\begin{tabular}{|l|l|l|}
\hline \multirow{2}{*}{ Transverse frontopolar gyrus } & left and right (dorsal) & Positive $t$-score \\
\cline { 2 - 3 } & right (ventral) & Negative $t$-score \\
\hline Lateral orbital frontal gyrus & Right & Negative $t$-score \\
\hline Superior frontal gyrus & right (frontal pole) & Negative $t$-score \\
\hline \multirow{2}{*}{ Superior frontal sulcus } & right (ventral) & Positive $t$-score \\
\cline { 2 - 3 } & right (dorsal) & Negative $t$-score \\
\hline \multirow{2}{*}{ Medial frontal gyrus } & right & Negative $t$-score \\
\hline Inferior frontal gyrus & right (caudal) & Positive $t$-score \\
\hline Inferior frontal sulcus & right (caudal) & Positive $t$-score \\
\hline Superior rostral gyrus & left & Positive $t$-score \\
\hline \multirow{2}{*}{ Cingulated sulcus } & caudal & Negative $t$-score \\
\cline { 2 - 3 } & caudal & Positive $t$-score \\
\hline
\end{tabular}




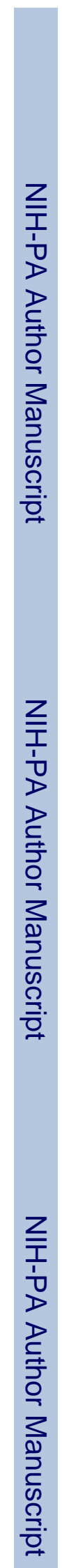

Goerke et al.

Page 26

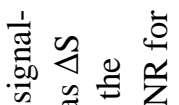

항

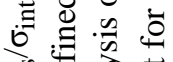

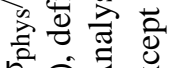

0

:

용

馬

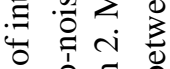

ป

离

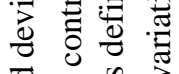

चै की

焉

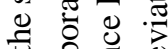

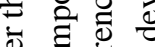

ठ ए ए

虫

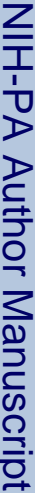

ส․ำ.

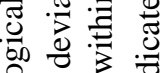

응 응 훙

爷焉离

पै के

官官

卷

ठ :

的的

츄 웜

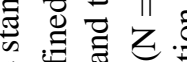

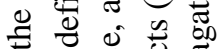

प응 응 응

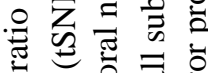

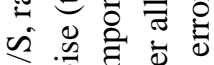

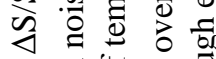

\& 즁

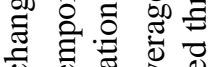

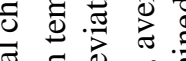

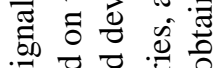

क D

: こี

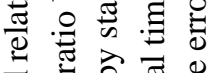

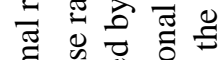

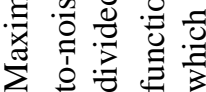

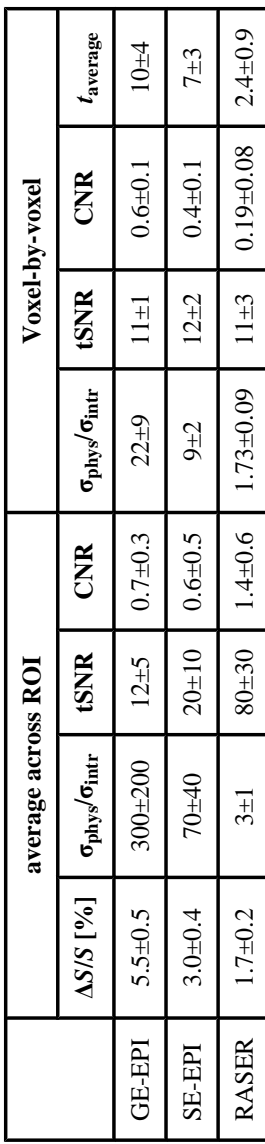

Neuroimage. Author manuscript; available in PMC 2012 January 1. 